\title{
QUALIDADE DE VIDA E TRABALHO DOCENTE NO ENSINO SUPERIOR: UMA RELAÇÃO ANTAGÔNICA
}

Quality of life and teaching work outside higher education: an antagonistic relationship

Calidad de vida y trabajo docente fuera de la educación superior: una relación antagónica

\section{Jeann Bruno Ferreira da Silva*1, Mônica Aparecida da Rocha Silva², Waldecy Rodrigues ${ }^{3}$.}

${ }^{1}$ Doutorando em Desenvolvimento Regional e Mestre em Ciências da Saúde pela Universidade Federal do Tocantins, Palmas, Brasil.

${ }^{1}$ Doutora em Ciências Sociais pela UnB, Docente lotada do Programa de Pós-graduação em Desenvolvimento Regional e no curso de graduação em Ciências Econômicas da Universidade Federal do Tocantins, Palmas, Brasil.

${ }^{1}$ Doutor em Estudos Comparados de Desenvolvimento pela UnB, Docente do Programa de Pós-graduação em Desenvolvimento Regional da Universidade Federal do Tocantins, Palmas, Brasil.

*Programa de Pós-graduação em Desenvolvimento Regional, Universidade Federal do Tocantins, Av. NS 15, 109 Norte, Palmas, Tocantins, Brasil. CEP:77.010-090. E-mail jbpsicologia@live.com.

\section{Artigo recebido em 14/02/2020 aprovado em 30/10/2020 publicado em 30/12/2020.}

\section{RESUMO}

Introdução: os docentes do ensino superior no Brasil além de lecionar aulas, dividem suas atribuições entre projetos de extensão, orientações de discentes e pesquisas acadêmicas. A crescente oferta de matrículas no ensino superior e a criação de novas universidades tem ampliado também a criação de programas de pósgraduação. Este processo, naturalmente capacita o docente para multifacetar sua atuação, seja por meio de programas de mestrado ou doutorado. Objetivo: analisar a produção científica acerca da Qualidade de Vida (QV) de Docentes do Ensino Superior. Metodologia: pesquisa bibliográfica de natureza descritiva com abordagem cienciométrica para análise e tratamento dos dados. Resultados e discussão: foram encontradas 78 publicações e, ao aplicar os critérios de inclusão e exclusão, restaram o total de 22. A maioria dos artigos (45\%) foram publicados no ano de $2015,36 \%$ foram publicados na região Sul, dos autores principais $54 \%$ possuem titulação de doutor, $45 \%$ objetivaram avaliar a QV dos profissionais docentes e as classificações de maior prevalência foram A2 e A1. Considerações finais: pontuou-se a escassez de pesquisas no sobre a temática na base de dados da SsciELO e que é necessário tornar visível o processo de trabalho destes profissionais para possíveis intervenções no âmbito universitário.

Palavras-chave: qualidade de vida; docente; ensino superior.

\section{ABSTRACT}

Introduction: higher education teachers in Brazil in addition to teaching classes divide their attributions between extension projects, student guidance and academic research. The increasing supply of enrollment in higher education and the creation of new universities has also expanded the creation of graduate programs. This process naturally empowers the teacher to multifacet his performance, either through master's or doctoral programs. Objective: to analyze the scientific production on quality of life (QoL) of Higher Education Teachers. Methodology: descriptive bibliographic research with a cienciometric approach for data analysis and treatment. Results and discussion: 78 publications were found and, when applying the inclusion and exclusion criteria, the total of 22 remained. Most articles (45\%) published in 2015, 36\% were published in the Southern region, of the main authors 54\% have a doctor's degree, $45 \%$ aimed to evaluate the Qol of teaching professionals and the highest prevalence classifications 
were A2 and A1. Final considerations: the scarcity of research on the subject was pointed out in the SsciELO database and that it is necessary to make visible the work process of these professionals for possible interventions in the university field.

Keywords: quality of life; teacher; faculty higher education.

\section{RESUMEN}

Introducción: los profesores de educación superior en Brasil, además de enseñar clases, dividen sus atribuciones entre proyectos de extensión, orientación estudiantil e investigación académica. La creciente oferta de matriculación en educación superior y la creación de nuevas universidades también ha ampliado la creación de programas de posgrado. Este proceso naturalmente faculta al maestro para multifacéticamente su desempeño, ya sea a través de programas de maestría o doctorado. Objetivo: analizar la producción científica sobre calidad de vida (QoL) de profesores de educación superior. Metodología: investigación bibliográfica descriptiva con un enfoque científicométrico para el análisis y tratamiento de datos. Resultados y discusión: se encontraron 78 publicaciones y, al aplicar los criterios de inclusión y exclusión, se mantuvo el total de 22. La mayoría de los artículos (45\%) publicado en 2015, 36\% fueron publicados en la región sur, de los principales autores 54\% tienen un título de médico, $45 \%$ destinado a evaluar el Qol de los profesionales de la enseñanza y las clasificaciones de mayor prevalencia fueron A2 y A1. Consideraciones finales: la escasez de investigación sobre el tema se señaló en la base de datos SsciELO y que es necesario hacer visible el proceso de trabajo de estos profesionales para posibles intervenciones en el ámbito universitario.

Descriptores: calidad de vida; maestro; facultad educación superior.

\section{INTRODUÇÃO}

O exercício do trabalho docente no ensino superior é considerado tamanha relevância científica e social, pois exige habilidades e competências que vão além da sala de aula, como elaboração de projetos de pesquisa, planejamento de aulas, participação em comissões, eventos, entre outros. Devido essa gama de fatores pertinentes à rotina laboral, este profissional pode apresentar suscetibilidade ao padecimento.

De fato, o trabalho é tido como basilar para a formação de indenitária do homem ocidental. Marx corroborou em seus escritos que o trabalho cria o homem, e que, por força da dialética, o homem cria a si mesmo pelo trabalho. Para ele o trabalho é a propriedade fundamental do homem, que é, em determinado grau, forjado pelo trabalho por ser, este, a sua possibilidade de transformar o mundo (MARX, 1998). Deste modo, infere-se que o trabalho constitui um elemento essencial para o homem, interferindo significativamente, também, para sua inserção na sociedade.

Outrossim, a carreira docente, antes percebida como segura, de elevado status social e econômico, vem ganhando outras configurações que impactam diretamente o profissional em si, como mudanças do ambiente, incluindo políticas e práticas organizacionais no tocante de flexibilização de atribuições (DE PAIVA \& SARAIVA, 2005; BOAS \& MORIN, 2014). Nesta perspectiva, professores-pesquisadores, que contam com vínculo formal e cujo trabalho envolve a combinação de docência, pesquisa e extensão, têm sido afetados pelo denominado produtivismo acadêmico, exigindo cada vez mais dedicação e esforço, além de promover uma competição excessiva, com impactos sobre sua 
saúde e bem-estar (NASCIMENTO \& SALVÁ, 2013).

Diante ao exposto, há de se considerar que todos estes fatores supramencionados incidem em vulnerabilidade emocional e, somados, influenciam na Qualidade de Vida - QV do profissional docente do ensino superior.

A Organização Mundial da Saúde define Qualidade de Vida como a percepção do indivíduo acerca de sua inserção na vida, no contexto da cultura e sistemas de valores nos quais ele vive e em relação aos seus objetivos, expectativas, padrões e preocupações, envolvendo bem estar espiritual, físico, mental, psicológico e emocional, além de relacionamentos sociais, como família e amigos e, também, saúde, educação, habitação saneamento básico e outras circunstâncias da vida (OMS, 2013).

Sob este enfoque, os aspectos inerentes à atividade docente no ensino superior trazem à tona o nível de sua qualidade de qualidade de vida, que também podem levar à prejuízos na relação professor-aluno e àqueles de cunho pedagógico, interferindo na qualidade do ensino.

Em consonância com o exposto, o estudo objetivou em analisar a produção científica, disponível na base de dados da Biblioteca Virtual da Scientific Electronic Library Online - SciELO acerca da Qualidade de Vida de profissionais Docentes do Ensino Superior.

Como questões norteadoras têm-se: 1 - As publicações indexadas à base de dados SciELO, em sua maioria, foram realizadas em quais regiões do país? 2 - Quais os anos de maior prevalência destas publicações? 3 - Qual titulação dos autores principais? 4 - Os estudos em questão possuem objetivo de avaliar apenas a Qualidade de Vida dos docentes do ensino superior?

\section{MATERIAIS E MÉTODOS}

A pesquisa caracteriza-se por ser bibliográfica de natureza descritiva, realizada entre os meses de abril a agosto do ano de 2019 e, para análise dos dados extraídos da bliblioteca virtual da SciELO, foi utilizada a técnica de Cienciometria.

Os estudos quantitativos acerca da produção científica permitem compreender a amplitude e a natureza das atividades de pesquisa desenvolvidas nas diferentes áreas do conhecimento, como o local, as instituições e a formação dos pesquisadores que o desenvolveram (NORONHA, 2000). Outro questão refere-se à difusão do conhecimento científico e o fluxo da informação sob enfoques múltiplos.

Cabe pontuar que a cienciometria não visa substituir quaisquer métodos analíticos sobre determinado assunto, mas sim, objetiva provocar maior visibilidade aos dados coletados na pesquisa. Tal recurso permite identificar quais áreas e/ou aspectos do fenômeno pesquisado necessitam de maior preocupação (LAURINDO \& MAFRA, 2010).

Justifica-se o uso da cienciometria na elaboração de políticas públicas pelo fato desta ser fundamental para a tomada de decisão no que tange a gestão de recursos de qualquer ordem, como no incentivo à geração de informações técnicas que permitam um desenvolvimento de fato sustentável. No caso do estudo em questão, especificamente sobre o enfoque à qualidade de 
vida do profissional docente do ensino superior no Brasil.

Os descritores utilizados delineados a partir da plataforma DeCS (Descritores em Ciências da Saúde), foram: QUALIDADE DE VIDA and DOCENTE and ENSINO SUPERIOR. Adotaram-se como critérios de inclusão: artigos em língua portuguesa, disponíveis em base de dados de acesso gratuito da SciELO, publicados nos últimos 10 (dez) anos, realizados no Brasil. Com critérios de exclusão, estiveram de fora: resumos, textos incompletos, estudos publicados em formatos de teses, dissertações e/ou monografias, artigos de cunho jornalístico, àqueles pulicados na íntegra em língua estrangeira e os que não possuem em seu teor um dos descritores DeCS utilizados.

Os resultados apontados neste estudo foram levantados a partir do estabelecimento de categorias. As categorias foram elencadas a partir dos seguintes dados de cada artigo: 1 - Ano de publicação do estudo; 2 - Região Geográfica em que o estudo foi realizado; 3 - Titulação do primeiro autor; 4 - Categoria/similaridade de objetivos do estudo e; 5 - Qualis do periódico conforme classificação da CAPES.

Para representação dos dados utilizou-se de gráficos, figuras e quadros, e, para a análise foi utilizada estatística descritiva simples, especificamente estabelecimento de percentuais, médias, prevalência e moda.

\section{RESULTADOS E DISCUSSÃO}

No total geral, utilizando os descritores "qualidade de vida" and "docente" and "ensino superior", foram encontradas 78 publicações na biblioteca da SciELO. Destas, as que apresentaram texto completo foram 40 , e, ao se aplicar os demais critérios de inclusão e exclusão, totalizaram 22 artigos, todos pesquisas aplicadas realizadas em campo. $\mathrm{O}$ ano da publicação dos referidos artigos estiveram entre os anos de 2009 e 2018. Os resultados são apresentados nos quadros abaixo:

Gráfico 1. Análise do Quantitativo e Ano de publicação dos periódicos

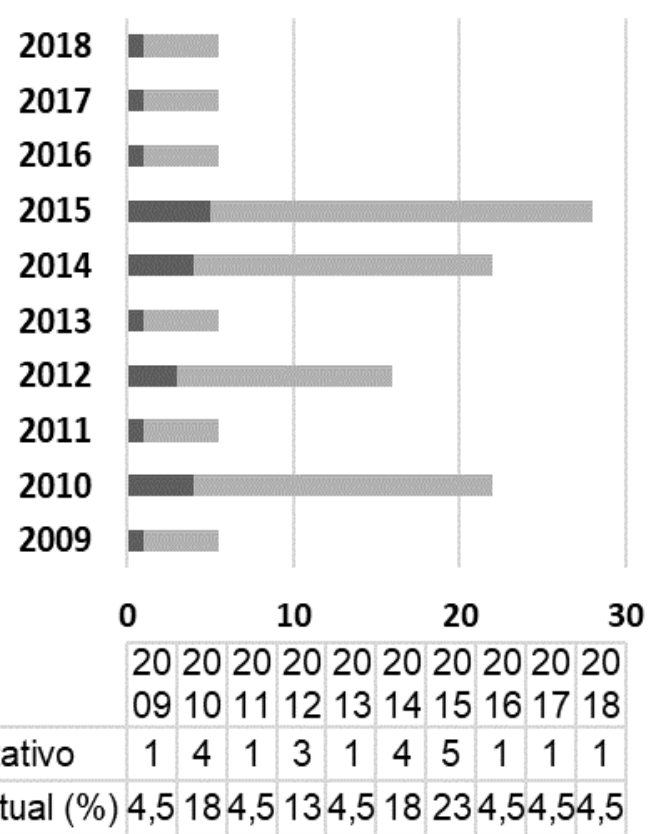

Fonte: dados da pesquisa.

Sobre o quantitativo de publicações por ano, destacou-se que 5 (23\%) foram correspondentes ao ano de 2015, seguido do ano de 2014 e 2010, cada uma com o quantitativo de 4 (18\%) publicações. A média das publicações totalizou 2,5 periódico por ano.

De acordo com o Censo da Educação Superior de 2015, realizado pelo Instituto Nacional de Estudos e Pesquisas Educacionais Anísio Teixeira - INEP 2,1\% das Instituições de Educação Superior (IES) ofereceram mais de 100 cursos de graduação. Neste mesmo ano mais de 
2,9 milhões de alunos ingressaram em cursos de educação superior de graduação. Outro fato que corroborou para os índices no referido ano devese ao número de docentes com doutorado, que cresceu tanto na rede pública, quanto na rede privada.

Aproximadamente $70 \%$ dos docentes nas universidades têm o regime de contrato de trabalho em tempo integral, bem superior aos docentes em tempo integral dos centros universitários $(25,8 \%)$ e faculdades $(18,7 \%)$. Quanto à Região Geográfica em que o periódico foi publicado, prevaleceu a região sul do Brasil publicação conforme periódico.

Imagem 1. Mapa do Brasil conforme Região Geográfica e percentual de publicação

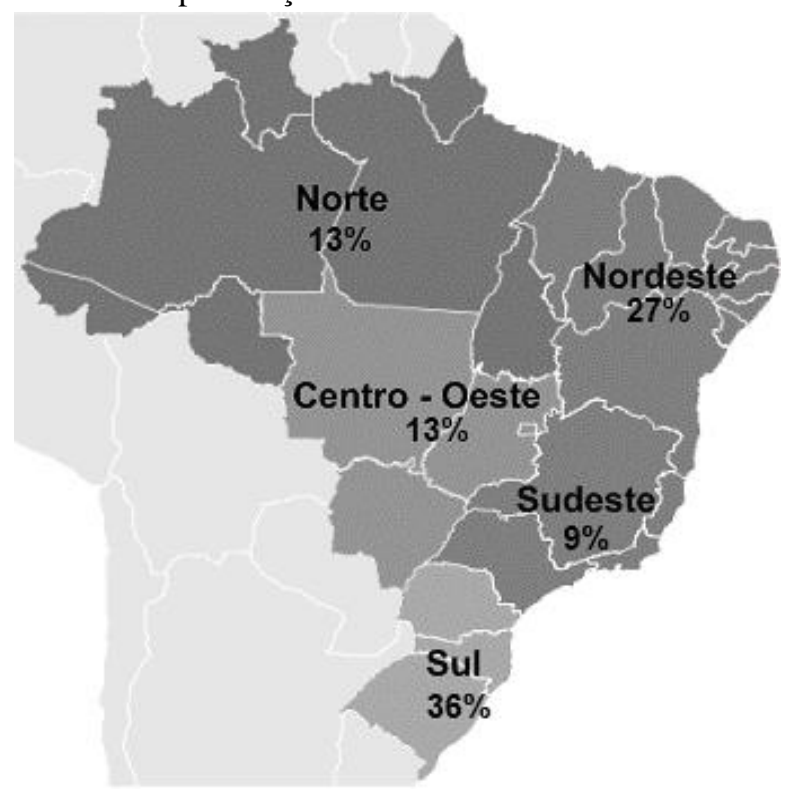

Fonte: dados da pesquisa.

Nesta busca na base de dados da SciELO, a região Sul apresentou prevalência de publicações, com total de $36 \%$ dos periódicos encontrados de acordo com os descritores préestabelecidos, enquanto a região Sudeste obteve o menor índice, representando $9 \%$.

Nesse quesito, convém abordar que a região Sul é favorecida no âmago da produção científica pela concentração de universidades e institutos de pesquisa já historicamente estabelecidos e consolidados.

Fator, este, que também evidencia o crescimento de recursos humanos docentes, contribuindo para o fenômeno adoecimento ocupacional.

Quadro 3. Titulação do primeiro autor

\begin{tabular}{|c|c|c|c|}
\hline Titulação & N. & Percentual & Moda \\
\cline { 1 - 3 } Graduado & 2 & $9 \%$ & \multirow{2}{*}{ Doutorado } \\
\cline { 1 - 3 } Mestrado & 8 & $36 \%$ & \\
\hline Doutorado & 12 & $54 \%$ & \\
\hline
\end{tabular}

Fonte: dados da pesquisa.

Do primeiro autor de cada publicação, aferiu-se que a maioria 12 (54\%) possuem titulação de doutor, seguido de 8 (36\%) mestres e, por último duas publicações cuja titulação do primeiro autor é apenas a Graduação (9\%).

Os doutores tendem a apresentar um perfil com maior direcionamento para a pesquisa e para a docência, enquanto que os mestres tendem a exercer papel na vida profissional ou na área privada, mostrando que há necessidade de criar medidas que incentivem os mestres a realizar o doutorado (VELLOSO, 2004).

Sobre as categorias dos objetivos mais prevalentes, destacou-se a categoria Avaliar a Qualidade de Vida de docentes do ensino superior, com total de 10 (45\%) das publicações e, por último, as categorias Constatar Benefícios sociais concedidos à docentes e Analisar Indicadores de $Q V$ de docentes, ambas com 1 artigo publicado cada, representando $4,5 \%$ das publicações. 
Quadro 4. Categoria/similaridade de objetivos do estudo

\begin{tabular}{|c|c|c|c|}
\hline $\begin{array}{c}\text { Categorias/similaridade de } \\
\text { objetivos }\end{array}$ & $\mathbf{N}^{\mathbf{o}}$ & $\%$ & Moda \\
\hline $\begin{array}{l}\text { Avaliar QV de docentes do } \\
\text { ensino superior }\end{array}$ & 10 & $45 \%$ & \multirow{6}{*}{$\begin{array}{c}\text { Avaliar } \\
\text { QV de } \\
\text { docentes } \\
\text { do } \\
\text { ensino } \\
\text { superior }\end{array}$} \\
\hline $\begin{array}{l}\text { Compreender a percepção } \\
\text { do docente sobre QV }\end{array}$ & 6 & $27 \%$ & \\
\hline $\begin{array}{l}\text { Verificar o impacto de uma } \\
\text { ação fonoaudiológica na QV } \\
\text { em voz de docentes }\end{array}$ & 2 & $9 \%$ & \\
\hline $\begin{array}{l}\text { Analisar a correlação entre } \\
\text { QV e estilo de vida }\end{array}$ & 2 & $9 \%$ & \\
\hline $\begin{array}{l}\text { Constatar Benefícios sociais } \\
\text { concedidos à docentes }\end{array}$ & 1 & $4,5 \%$ & \\
\hline $\begin{array}{l}\text { Analisar Indicadores de QV } \\
\text { de docentes }\end{array}$ & 1 & $4,5 \%$ & \\
\hline
\end{tabular}

Fonte: dados da pesquisa.

Quadro 5. Qualis do periódico conforme classificação da CAPES (interdisciplinar)

\begin{tabular}{|c|c|c|}
\hline PERIÓDICO & QUALIS & ÁREA \\
\hline Cien. \& Saúde Coletiva & A1 & SOCIOLOGIA \\
\hline Cien. \& trabajo & B1 & EDUCAÇÃO \\
\hline $\begin{array}{l}\text { Trabalho, Educação e } \\
\text { Saúde }\end{array}$ & $\mathrm{A} 2$ & EDUCAÇÃO \\
\hline P. Escolar e Educacional & A1 & ENSINO \\
\hline Revista CEFAC & $\mathrm{A} 2$ & $\begin{array}{l}\text { LINGUÍSTICA E } \\
\text { LITERATURA }\end{array}$ \\
\hline Rev. Bras. Epidemio. & B1 & $\begin{array}{l}\text { CIÊNCIAS } \\
\text { AMBIENTAIS }\end{array}$ \\
\hline Educação e Sociedade & A1 & EDUCAÇÃO \\
\hline $\begin{array}{l}\text { Trabalho, Educação e } \\
\text { Saúde }\end{array}$ & A2 & EDUCAÇÃO \\
\hline $\begin{array}{l}\text { Avaliação: Rev. da Av. da } \\
\text { Educacão Superior }\end{array}$ & A1 & EDUCAÇÃO \\
\hline Revista CEFAC & A2 & $\begin{array}{l}\text { LINGUÍSTICA E } \\
\text { LITERATURA }\end{array}$ \\
\hline Revista de Salud Pública & A1 & ENSINO \\
\hline Revista CEFAC & A2 & $\begin{array}{l}\text { LINGUÍSTICA E } \\
\text { LITERATURA }\end{array}$ \\
\hline Rev. Ed. Física/UEM & A2 & $\begin{array}{l}\text { ARQUITETURA, } \\
\text { URBANISMO E } \\
\text { DESIGN }\end{array}$ \\
\hline Escola Ana Nery & A2 & ENSINO \\
\hline Trab., Educação e Saúde & A2 & EDUCAÇÃO \\
\hline Cien. \& Saúde Coletiva & A1 & SOCIOLOGIA \\
\hline Rev. de Enf. Referência & $\mathrm{B} 2$ & ENFERMAGEM \\
\hline Motriz: Rev. de Ed. Fís. & A2 & $\begin{array}{l}\text { ARQ. URB. E } \\
\text { DESIGN }\end{array}$ \\
\hline $\begin{array}{lrr}\text { Rev. Bras. } & \text { de } \\
\text { Cineantropometria } & \text { e } \\
\text { Desempenho Humano } & \\
\end{array}$ & B1 & $\begin{array}{l}\text { CIÊNCIAS } \\
\text { AMBIENTAIS }\end{array}$ \\
\hline Motricidade & $\mathrm{B} 1$ & EDUCAÇÃO \\
\hline Rev. Bras. Ed. Médica & B1 & EDUCAÇÃO \\
\hline Educar em Revista & B1 & ARTES \\
\hline
\end{tabular}

Fonte: dados da pesquisa.
Sobre este aspecto, a avaliação da QV de docentes é caracterizada como um fenômeno que necessita de intervenção emergencial, que tem ganhado maior visibilidade no universo de problemas em que a ciência tem buscado compreender. Dado este, evidenciado por meio da técnica de cienciometria. No que se refere à classificação Qualis/CAPES das referidas publicações, o quadro abaixo caracteriza cada periódico.

As classificações de maior prevalência foram A2 e A1, conotando relevância e qualidade de impacto. Assim, as pesquisas sobre QV de docentes do ensino superior no Brasil também tem ganhado visibilidade internacional.

\section{CONCLUSÃO}

Conclui-se que, as publicações que abordam sobre a Qualidade de Vida de docentes do ensino superior emergem que a profissão tem ganhado outras configurações que vão além lecionar. Destaca-se que paralelo a esta última, a atribuição de pesquisador e extensionista também tem exigido um desdobramento e um misto de funções que tem corroboram para este padecimento.

Em resposta às questões norteadoras, as publicações indexadas à base de dados SciELO, em sua maioria, foram realizadas na região Sul do país, sendo o ano de 2015 o que apresenta maior prevalência destas publicações. Quanto titulação dos autores principais, a maioria possui doutorado. Também evidenciou-se que a maioria dos estudos em questão possui objetivo de avaliar Qualidade de Vida dos docentes do ensino superior. 
Por meio deste estudo, evidenciou-se a necessidade de se realizar mais pesquisas sobre o assunto em questão, bem como conferir maior relevância ao fenômeno Qualidade de Vida dos profissionais docentes do ensino superior e dar maior visibilidade sobre processo de trabalho destes profissionais.

Além da análise cienciométrica da base de dados da SciELO, destacou-se que os dados bibliográficos utilizados deram ênfase no processo de trabalho e no comprometimento que esta gama de atribuições designadas aos docentes exercem sua qualidade de vida, podendo levar ao padecimento e contribuir para o absenteísmo e afastamentos para tratamento de saúde. Este fato além de criar um espectro mórbido à universidade, também pode gerar ônus na contratação de profissionais substitutos.

Este estudo possuiu limitações, estas se dão pelo motivo de ter sido consolidado apenas uma base de dados. Portanto, sugere-se que as futuras pesquisas sobre o tema sejam realizadas de maneira interdisciplinar em outras bases de dados, sejam públicos ou privados, que abordem sobre qual influência que gestão acadêmica/universitária possui neste processo. Para que se amplie o panorama acerca da produção científica da Qualidade de Vida dos profissionais docentes do ensino superior no Brasil, também propõe-se que se realizem novos estudos baseados nas necessidades e políticas públicas de cada região do Brasil. Assim será possível compreender geograficamente o mapa deste fenômeno que a cada dia está mais crescente também em outras categorias profissionais.

\section{AGRADECIMENTO}

Agradecemos à Coordenação do Programa de Pós-Graduação em Desenvolvimento Regional (PPGDR) da Universidade Federal do Tocantins, por oportunizar espaços para a produção de conhecimento sobre a relação entre Universidade e o Desenvolvimento Regional.

Todos os autores declararam não haver qualquer potencial conflito de interesses referente a este artigo.

\section{REFERÊNCIAS}

ANCHIETA, Vânia Cristine Cavalcante e colaboradores. Trabalho e riscos de adoecimento: um estudo entre policiais civis. Psicologia: Teoria e Pesquisa, v. 27, n. 2, p. 199-208, 2011.

BOAS, Ana Alice Vilas; MORIN, Estelle M. Sentido do trabalho e fatores de qualidade de vida no trabalho: a percepção de professores brasileiros e canadenses. Revista Alcance, v. 23, n. 3, p. 272292, 2016.

DE PAIVA, Kely César Martins; SARAIVA, Luiz Alex Silva. Estresse ocupacional de docentes do ensino superior. Revista de AdministraçãoRAUSP, v. 40, n. 2, p. 145-158, 2005.

IRIGARAY, Hélio Arthur R. VÍNCULOS PROFISSIONAIS E SENTIDO DO TRABALHO: UMA PESQUISA COM PROFESSORES DO ENSINO SUPERIOR. Revista de Administração Mackenzie, v. 20, n. 1, p. 1-27, 2019.

KLEIN, Karla Barbosa. Qualidade de vida no trabalho docente: um olhar sobre os professores da Universidade Federal do Tocantins. 2013.

INEP. Instituto Nacional de Estudos e Pesquisas Educacionais Anísio Teixeira. Notas Estatísticas Censo da Educação Superior 2015. Brasília, 2015.

LAURINDO, R.; MAFRA, T. Cienciometria da revista Comunicação \& Sociedade identifica interfaces da área, Comunicação \& Sociedade, n. 53, p. 233-260, jan./jun. 2010. 
MARX, Karl. O capital, livro I, vol. 1. Rio de Janeiro: Civilização Brasileira, 1998.

MEDEIROS, Ana Lúcia; TEIXEIRA, Maria Luisa Mendes. Limites da dignidade de docentes nas práticas de gestão em universidades brasileiras. Revista Gestão Universitária na América Latina-GUAL, v. 10, n. 2, p. 134-154, 2017.

MENEZES, LM de; NEPOMUCENO, L. H.; BATISTA-DOS-SANTOS, A. C. Os sentidos do trabalho para um grupo de professores de uma universidade pública: A dialética prazersofrimento em tempos de flexibilidade. Anais EnANPAD, 2011.

MORETTI, S. Qualidade de vida no trabalho x auto-realização humana. Florianópolis: Instituto Catarinense de Pós-Graduação, 2005.
NASCIMENTO, Rejane Prevot; SALVÁ, Maria Nair Rodrigues. A política de avaliação da pósgraduação Stricto-Sensu e o Trabalho Docente: Rumo ao "Produtivismo Acadêmico. Artigo apresentado no IV Encontro de Gestão de Pessoas e Relações de Trabalho, 2013.

NORONHA, D.P. Produção científica: análise cienciométrica das comunicações apresentadas nos SNBUs 1978-1998. In: XI Seminário Nacional de Bibliotecas Universitárias, 2000, Florianópolis, SC. Anais... Florianópolis: UFSCBU, 2000. p. 1-12.

ORGANIZAÇÃO MUNDIAL DA SAÚDE. Qualidade de vida em 5 passos. Biblioteca Virtual em Saúde: Dicas em Saúde, 2013. Disponível em: https://bvsms.saude.gov.br/bvs/dicas/260_qualid ade_de_vida.html. Acesso em 18 jun 2019. 